\title{
An Effective Synthesis of 5,4'-Disubstituted Flavones via a Cesium Enolate Assisted Intramolecular ipso-Substitution Reaction
}

\author{
Masato Matsugi,* Masaki Takeda, Ayano Takahashi, Takahide TAZaki, Hiroto Tamura, and \\ Takayuki SHIOIRI \\ Department of Applied Biological Chemistry, Faculty of Agriculture, Meijo University; 1-501 Shiogamaguchi, \\ Tempaku-ku, Nagoya 468-8502, Japan. $\quad$ Received April 16, 2010; accepted May 29, 2010; published online June 1, 2010
}

\begin{abstract}
A variety of 5,4'-disubstituted flavones, which are anticipated to be androgen receptor antagonists to treat diseases mediated by the androgen receptor, were synthesized. It was found that an intramolecular ipso-substitution reaction via cesium enolate using 2-fluoro-6-hydroxyacetophenone and various benzoyl chlorides was effective in the preparation of 5-hydroxy-4'-alkylflavones.
\end{abstract}

Key words flavone; ipso-substitution; androgen; antagonist

The androgen receptor (AR), which is activated by androgenic hormones, functions as a DNA binding transcription factor that regulates gene expression. ${ }^{1,2)}$ The androgen-regulated genes are important for the development and maintenance of the male sexual phenotype. It is known that isoflavones are similar to the estrogen hormone, and several studies using flavones as AR antagonists have been reported. ${ }^{3,4)}$ We have already reported that some flavonoids act as a novel androgen receptor ligand which interacts with the receptor in a different manner from known androgen receptor ligands. ${ }^{5)}$ We also disclosed that 5-hydroxyflavone have extremely high AR antagonistic activity, and that the hydroxyl group at the 5-position plays an important role in the interaction with the AR. Furthermore, the introduction of hydroxyl group at the 4'-position increases AR antagonistic activity as well. We report herein the syntheses of various 5,4'-disubstituted flavones, which are expected to be AR antagonist, using a novel intramolecular ipso-substitution method to construct the flavone-skeleton.

\section{Results and Discussion}

We planned to prepare a number of 5,4'-disubstituted flavones, shown in Table 1, and assay them for AR antagonistic activity. The compounds with asterisk are novel flavones and the others are flavones, with unknown AR antagonistic activity. These compounds were chosen because of the variation in the substituent constant ${ }^{(6)}$ and STERIMOL parameter $^{7,8)}$ of the $4^{\prime}$-position substituent as shown in Table 2.

At the outset, 5 -hydroxyflavones $(\mathbf{3 a}-\mathbf{h})$ in Table 3 were prepared according to the known method $\left.{ }^{9}\right)$ using 2,6-dihydroxyacetophenone and the corresponding benzoyl chlorides under basic conditions in acetone. ${ }^{10}$ The yields were low except for the 5-hydroxy-4'-trifluoromethylflavone (3e). The ${ }^{1} \mathrm{H}-\mathrm{NMR}$ of the crude products showed significant contamination with by-products. More importantly, the desired reaction did not proceed at all, when 4-alkylbenzoyl chlorides were used as substrate (Table 1, entries 9, 10). In this case, the major product was $\mathbf{4}$ even under high dilution conditions (Fig. 1). ${ }^{11)}$

Thus finding a new method to prepare $4^{\prime}$-alkylflavones was imperative. To prevent the double benzoylation of the alkylbenzoyl chloride to 2,6-dihydroxyacetophenone, 2fluoro-6-hydroxyacetophenone $\mathbf{5}$ was used as an alternative substrate. We hypothesized that the electron-withdrawing effect of the fluoro-group on the aromatic ring would decrease the electron density of the corresponding phenoxy anion intermediate. And the various 5-hydroxyflavones would be

Table 1. Flavones Pointing for AR Antagonist

\begin{tabular}{lll|cll}
\hline \hline Entry & $\mathrm{R}^{1}$ & \multicolumn{1}{c|}{$\mathrm{R}^{2}$} & Entry & $\mathrm{R}^{1}$ & $\mathrm{R}^{2}$ \\
\hline 1 & $\mathrm{OH}$ & $\mathrm{F}$ & 10 & $\mathrm{OH}$ & $t \mathrm{Bu}$ \\
2 & $\mathrm{OH}$ & $\mathrm{Cl}$ & $11^{a)}$ & $\mathrm{OH}$ & $\mathrm{NH}_{2}$ \\
$3^{a}$ & $\mathrm{OH}$ & $\mathrm{CN}$ & $\left.12^{a}\right)$ & $\mathrm{OH}$ & $\mathrm{NHAc}^{a}$ \\
4 & $\mathrm{OH}$ & $\mathrm{NO}_{2}$ & $13^{a}$ & $\mathrm{OH}$ & $\mathrm{NAc}_{2}$ \\
$5^{a}$ & $\mathrm{OH}$ & $\mathrm{CF}_{3}$ & 14 & $\mathrm{OMe}$ & $\mathrm{F}$ \\
$6^{a}$ & $\mathrm{OH}$ & $\mathrm{SMe}^{a}$ & 15 & $\mathrm{OMe}$ & $\mathrm{CF}_{3}$ \\
$7^{a}$ & $\mathrm{OH}$ & $\mathrm{NMe}_{2}$ & $16^{a)}$ & $\mathrm{F}$ & $\mathrm{F}$ \\
8 & $\mathrm{OH}$ & $\mathrm{Ph}$ & $17^{a)}$ & $\mathrm{F}$ & $\mathrm{Cl}$ \\
9 & $\mathrm{OH}$ & $\mathrm{Me}$ & $18^{a)}$ & $\mathrm{F}$ & $\mathrm{CN}$ \\
\hline
\end{tabular}

a) Novel flavones.

Table 2. Substituent Constant ${ }^{4)}$ and STERIMOL Parameter ${ }^{5)}$

\begin{tabular}{lcc|lcc}
\hline \hline $\mathrm{R}^{2}$ & $\begin{array}{c}\text { Substituent } \\
\text { constants }(\sigma)\end{array}$ & STERIMOL & \multicolumn{1}{|c}{$\mathrm{R}^{2}$} & $\begin{array}{c}\text { Substituent } \\
\text { constants }(\sigma)\end{array}$ & STERIMOL \\
\hline $\mathrm{NMe}_{2}$ & -0.83 & 3.53 & $\mathrm{SMe}$ & 0 & 4.3 \\
$\mathrm{NH}_{2}$ & -0.66 & 2.93 & NHAc & 0 & 5.15 \\
$\mathrm{OH}$ & -0.37 & 2.74 & $\mathrm{NAc}$ & 0 & 7.15 \\
$\mathrm{OMe}$ & -0.27 & 3.98 & $\mathrm{~F}$ & 0.06 & 2.65 \\
$t \mathrm{Bu}$ & -0.2 & 4.11 & $\mathrm{Cl}$ & 0.23 & 4.3 \\
$\mathrm{Me}$ & -0.17 & 3 & $\mathrm{CF}_{3}$ & 0.54 & 3.3 \\
$\mathrm{Ph}$ & -0.01 & - & $\mathrm{CN}^{2}$ & 0.66 & 4.23 \\
$\mathrm{H}$ & 0 & 2.06 & $\mathrm{NO}_{2}$ & 0.78 & 3.44 \\
& & & & &
\end{tabular}

Table 3. Synthesis of 5-Hydroxyflavones $(\mathbf{3 a}-\mathbf{h})$

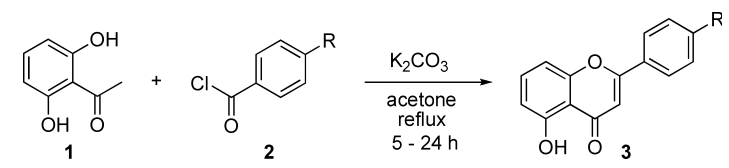

\begin{tabular}{|c|c|c|c|c|c|}
\hline Entry & $\mathrm{R}$ & Yield $(\%)^{a)}$ & Entry & $\mathrm{R}$ & Yield $(\%)^{a)}$ \\
\hline 1 & $\mathrm{~F}: \mathbf{3 a}$ & 22 & 6 & SMe: $3 f$ & 9 \\
\hline 2 & $\mathrm{Cl}: \mathbf{3 b}$ & 23 & 7 & $\mathrm{NMe}_{2}: \mathbf{3 g}$ & 10 \\
\hline 3 & $\mathrm{CN}: \mathbf{3 c}$ & 34 & 8 & $\mathrm{Ph}: \mathbf{3 h}$ & 15 \\
\hline 4 & $\mathrm{NO}_{2}: \mathbf{3 d}$ & 37 & 9 & Me: 3i & $0^{b)}$ \\
\hline 5 & $\mathrm{CF}_{3}: \mathbf{3 e}$ & 78 & 10 & $t \mathrm{Bu}: \mathbf{3 j}$ & $0^{c)}$ \\
\hline
\end{tabular}

a) Isolated yield after recrystallization from ethyl acetate. $b$ ) The major product was $\mathbf{4 i}$. c) The major product was $\mathbf{4 j}$ 
obtained via the plausible mechanism shown in Fig. 2, if an intramolecular ipso-substitution reaction ${ }^{12)}$ of an enolate proceeds.

Although there had been no report of flavone synthesis using intramolecular ipso-substitution reaction, we found that the treatment of 2-fluoro-6-hydroxyacetophenone 5, benzoyl chloride $\mathbf{2 k}$, and potassium carbonate provided 5-hydroxyflavone in good yield ( $78 \%$ recryst. from ethyl acetate) (Table 4, entry 1). After exploring a variety of reaction conditions, we found that cesium carbonate was better as the base than other carbonates $\left(\mathrm{Li}_{2} \mathrm{CO}_{3}, \mathrm{Na}_{2} \mathrm{CO}_{3}\right.$, and $\left.\mathrm{K}_{2} \mathrm{CO}_{3}\right)$ in terms of reaction rate, and either acetone or $N, N$-dimethylformamide (DMF) was suitable as solvent. We then tried to prepare 5-hydroxyflavones (3i, 3j, 3f, 3g), which were difficult to obtain in good yields with the reported method. ${ }^{9}$ The yields were improved as shown in Table 4 and enough

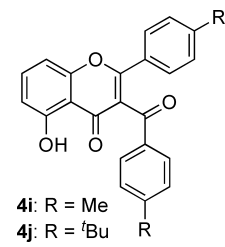

Fig. 1. By-products Dibenzoylated amount of the target flavones were obtained for the assay. We believe that the ipso-substitution method will be one of the most effective synthetic methods for various 5-hydroxyflavones, if suitable substrates are available.

Attempts to utilize the intramolecular ipso-substitution method to prepare 5-fluoroflavones proved futile. Therefore we had to apply the known synthetic method ${ }^{13,14)}$ to prepare the novel 5-fluoroflavones $(\mathbf{7 a - c})$ (Fig. 3). Similarly, 5methoxyflavones $(\mathbf{1 0 a}, \mathbf{b})$ were also prepared using another known method ${ }^{15)}$ because the corresponding substrates for the intramolecular ipso-substituted protocol were difficult to obtain (Fig. 4).

Finally, novel 5-hydroxy-4'-aminoflavone derivatives (3In) were prepared from 5-hydroxy-4'-nitroflavone (3d) as shown in Fig. 5. The prepared novel flavones (3c, 3e, 3f, 3g, 3l-n) and the other flavones, which have not been examined for AR antagonist activity $\left(\mathbf{3 a},{ }^{9)} \mathbf{3 b},{ }^{16)} \mathbf{3} \mathbf{d},{ }^{9)} \mathbf{3 h},{ }^{17)} \mathbf{3} \mathbf{i},{ }^{16)} \mathbf{3} \mathbf{j},{ }^{18)}\right.$ $\mathbf{3} \mathbf{k},{ }^{19)} \mathbf{1 0 a},{ }^{20)} \mathbf{1 0 \mathbf { b } ^ { 2 0 ) }}$ ), will be tested in an in-vitro reporter gene assay in the near future.

\section{Experimental}

Melting points are uncorrected. Infrared absorption spectra were recorded on a Bibby Scientific Ltd. Stuart ${ }^{\mathbb{B}}$ SMP $30 .{ }^{1} \mathrm{H}-\mathrm{NMR}$ spectra were measured in $\mathrm{CDCl}_{3}$ on JNM-EX270 $(270 \mathrm{MHz})$ spectrometers with tetramethylsilane as the internal standard. Mass spectra were recorded on a Shimadzu Corp.,

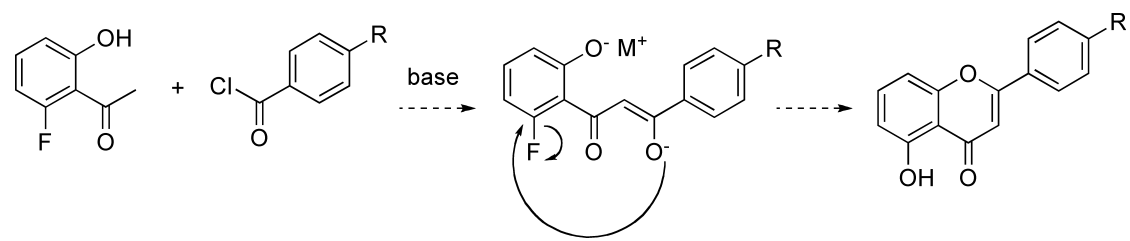

Fig. 2. Synthetic Strategy for Flavone Synthesis via Intramolecular ipso-Substitution of an Enolate

Table 4. Flavone Synthesis via Intramolecular ipso-Substitution

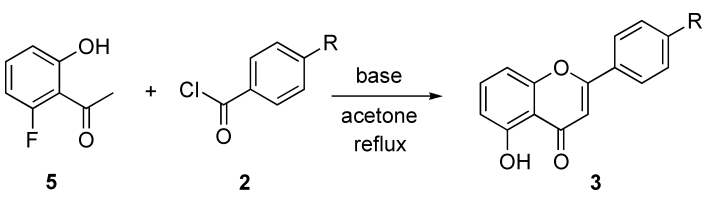

\begin{tabular}{cllcc}
\hline \hline Entry & \multicolumn{1}{c}{$\mathrm{R}$} & Base & Time (h) & Yield (\%) \\
\hline 1 & $\mathrm{H}: \mathbf{3 k}$ & $\mathrm{K}_{2} \mathrm{CO}_{3}$ & 24 & $78^{a)}$ \\
2 & $\mathrm{Me}: \mathbf{3 i}$ & $\mathrm{Cs}_{2} \mathrm{CO}_{3}$ & 3 & $68^{b)}$ \\
3 & $\mathrm{CN}: \mathbf{3 c}$ & $\mathrm{Cs}_{2} \mathrm{CO}_{3}$ & 4 & $68^{a)}$ \\
4 & ${ }^{t} \mathrm{Bu}: \mathbf{3 j}$ & $\mathrm{Cs}_{2} \mathrm{CO}_{3}$ & 2 & $54^{b)}$ \\
5 & $\mathrm{SMe}: \mathbf{3 f}$ & $\mathrm{Cs}_{2} \mathrm{CO}_{3}$ & 3 & $44^{b)}$ \\
6 & $\mathrm{NMe}_{2}: \mathbf{3 g}$ & $\mathrm{Cs}_{2} \mathrm{CO}_{3}$ & 4 & $47^{b)}$
\end{tabular}

a) Isolated yield after recrystallization from ethyl acetate. b) Isolated yield after column chromatography.

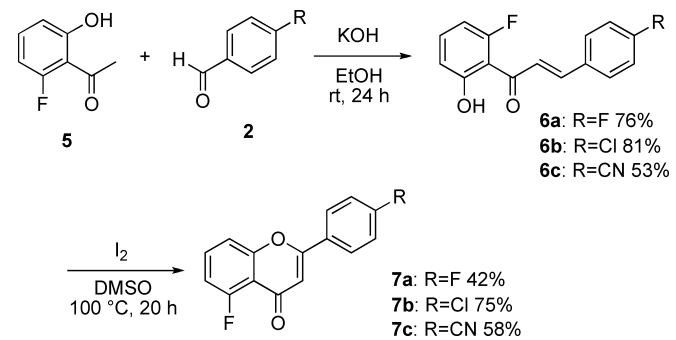

Fig. 3. Synthesis of Novel 5-Fluoroflavones

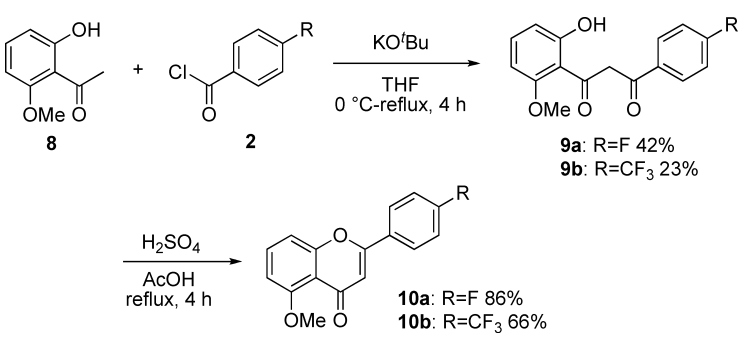

Fig. 4. Synthesis of 5-Methoxyflavones, Which Were Not Examined for AR Antagonist

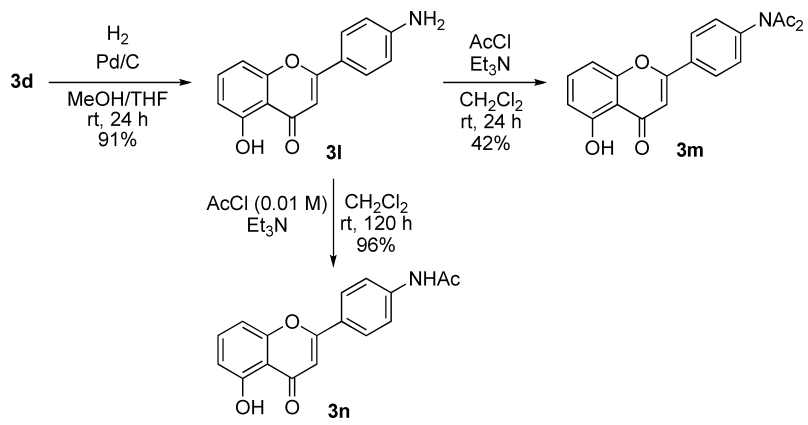

Fig. 5. Synthesis of Novel 5-Hydroxy-4'-aminoflavone Derivatives 
Ltd. QP5050A mass spectrometer. Wakogel ${ }^{\circledR}$ C-300 (45-75 $\left.\mu \mathrm{m}\right)$ was used for column chromatography and flash column chromatography. The chemicals used, were purchased from Sigma-Aldrich Co., Wako Pure Chemical Industries, Ltd., Tokyo Chemical Industry Co., Ltd., and Apollo Scientific Ltd. companies.

Typical Procedure for the Intramolecular ipso-Substitution Reaction To a stirred suspension of 2-fluoro-6-hydroxyacetophenone $\mathbf{5}(98 \mathrm{mg}$, $0.64 \mathrm{mmol}$ ) and 4-methylbenzoyl chloride $2 \mathbf{i}(120 \mathrm{mg}, 0.78 \mathrm{mmol})$ in acetone $(10 \mathrm{ml})$, was added cesium carbonate $(635 \mathrm{mg}, 1.95 \mathrm{mmol})$ at room temperature. The reaction mixture was stirred at $70^{\circ} \mathrm{C}$ for $3 \mathrm{~h}$, and quenched with $1 \mathrm{M} \mathrm{HCl}$, then extracted with AcOEt. The organic layer was washed with brine, dried with sodium sulfate and concentrated in vacuo. The residue was purified by recrystallization from ethyl acetate to give 5-hydroxy-4'methylflavone $3 \mathbf{i}^{16)}(110 \mathrm{mg}, 68 \%$ yield $)$ as yellow crystals; $\mathrm{mp} 172.0$ $173.0^{\circ} \mathrm{C} .{ }^{1} \mathrm{H}-\mathrm{NMR} \delta: 2.45(3 \mathrm{H}, \mathrm{s}), 6.71(1 \mathrm{H}, \mathrm{s}), 6.81(1 \mathrm{H}, \mathrm{d}, J=7.6 \mathrm{~Hz})$, $7.00(1 \mathrm{H}, \mathrm{d}, J=7.8 \mathrm{~Hz}), 7.31(2 \mathrm{H}, \mathrm{d}, J=8.6 \mathrm{~Hz}), 7.54(1 \mathrm{H}, \mathrm{t}, J=8.4 \mathrm{~Hz}), 7.82$ $(2 \mathrm{H}, \mathrm{d}, J=8.6 \mathrm{~Hz}), 12.62(1 \mathrm{H}, \mathrm{s})$. MS $m / z: 252\left(\mathrm{M}^{+}\right), 136$.

5-Hydroxy-4'-fluoroflavone $\mathbf{3 a}^{9)}$ Yellow crystals; mp $158.0-160.0^{\circ} \mathrm{C}$. ${ }^{1} \mathrm{H}-\mathrm{NMR} \delta$ : $6.69(1 \mathrm{H}, \mathrm{s}), 6.83(1 \mathrm{H}, \mathrm{d}, J=8.1 \mathrm{~Hz}), 7.00(1 \mathrm{H}, \mathrm{d}, J=10.8 \mathrm{~Hz})$, $7.22(2 \mathrm{H}, \mathrm{d}, J=8.1 \mathrm{~Hz}), 7.56(1 \mathrm{H}, \mathrm{t}, J=8.1 \mathrm{~Hz}), 7.91-7.96(2 \mathrm{H}, \mathrm{m}), 12.53$ $(1 \mathrm{H}, \mathrm{s})$. Anal. Calcd for $\mathrm{C}_{15} \mathrm{H}_{9} \mathrm{O}_{3} \mathrm{~F}: \mathrm{C}, 70.31 ; \mathrm{H}, 3.54$. Found: C, 70.41; H, 3.73 .

5-Hydroxy-4'-chloroflavone $\mathbf{3 b}^{16)}$ Yellow crystals; mp 175.0 $176.0^{\circ} \mathrm{C} .{ }^{1} \mathrm{H}-\mathrm{NMR} \delta: 6.72(1 \mathrm{H}, \mathrm{s}), 6.84(1 \mathrm{H}, \mathrm{d}, J=7.6 \mathrm{~Hz}), 6.92(1 \mathrm{H}, \mathrm{m})$, $7.49-7.60(3 \mathrm{H}, \mathrm{m}), 7.84-7.89(2 \mathrm{H}, \mathrm{m}), 12.50(1 \mathrm{H}, \mathrm{s}) . \mathrm{MS} m / z: 272\left(\mathrm{M}^{+}\right)$, $274\left(\mathrm{M}^{+}\right)+2,136,108$.

5-Hydroxy-4'-cyanoflavone 3c Yellow crystals; mp 195.0-196.0 ${ }^{\circ} \mathrm{C}$. ${ }^{1} \mathrm{H}-\mathrm{NMR} \delta: 6.79(1 \mathrm{H}, \mathrm{s}), 6.86(1 \mathrm{H}, \mathrm{d}, J=8.4 \mathrm{~Hz}), 7.03(1 \mathrm{H}, \mathrm{d}, J=8.1 \mathrm{~Hz})$, $7.60(1 \mathrm{H}, \mathrm{t}, J=8.1 \mathrm{~Hz}), 7.85(2 \mathrm{H}, \mathrm{d}, J=8.1 \mathrm{~Hz}), 8.04(2 \mathrm{H}, \mathrm{d}, J=8.1 \mathrm{~Hz})$, $12.37(1 \mathrm{H}, \mathrm{s})$. MS $m / z: 263\left(\mathrm{M}^{+}\right), 136,108$. Anal. Calcd for $\mathrm{C}_{16} \mathrm{H}_{9} \mathrm{O}_{3} \mathrm{~N}: \mathrm{C}$, 73.00; H, 3.45; N, 5.32. Found: C, 72.64; H, 3.41; N, 5.28.

5-Hydroxy-4'-nitroflavone $\mathbf{3 d ^ { 9 }}$ Yellow crystals; $m p$ 226.0-227.0 ${ }^{\circ} \mathrm{C}$. ${ }^{1} \mathrm{H}-\mathrm{NMR} \delta: 6.83(1 \mathrm{H}, \mathrm{s}), 6.88(1 \mathrm{H}, \mathrm{d}, J=8.1 \mathrm{~Hz}), 7.04(1 \mathrm{H}, \mathrm{d}, J=7.6 \mathrm{~Hz})$, $7.61(1 \mathrm{H}, \mathrm{t}, J=8.1 \mathrm{~Hz}), 8.10(2 \mathrm{H}, \mathrm{d}, J=7.0 \mathrm{~Hz}), 8.40(2 \mathrm{H}, \mathrm{d}, J=7.0 \mathrm{~Hz})$, $12.34(1 \mathrm{H}, \mathrm{s})$. MS $m / z: 283\left(\mathrm{M}^{+}\right), 237,108$.

5-Hydroxy-4'-trifluoromethylflavone 3e Yellow crystals; mp 138.0$139.0{ }^{\circ} \mathrm{C} .{ }^{1} \mathrm{H}-\mathrm{NMR} \delta: 6.79(1 \mathrm{H}, \mathrm{s}), 6.85(1 \mathrm{H}, \mathrm{d}, J=8.1 \mathrm{~Hz}), 7.03(1 \mathrm{H}, \mathrm{d}, J=$ $10.8 \mathrm{~Hz}), 7.59(1 \mathrm{H}, \mathrm{t}, J=8.1 \mathrm{~Hz}), 7.81(2 \mathrm{H}, \mathrm{d}, J=8.1 \mathrm{~Hz}), 8.04(2 \mathrm{H}, \mathrm{d}, J=$ $8.1 \mathrm{~Hz}), 12.43(1 \mathrm{H}, \mathrm{s})$. Anal. Calcd for $\mathrm{C}_{16} \mathrm{H}_{9} \mathrm{O}_{3} \mathrm{~F}_{3}: \mathrm{C}, 62.75 ; \mathrm{H}, 2.96$. Found: C, $62.81 ; \mathrm{H}, 3.09$.

5-Hydroxy-4'-methylthioflavone 3f Yellow crystals; mp 166.0$167.0^{\circ} \mathrm{C} .{ }^{1} \mathrm{H}-\mathrm{NMR} \delta: 2.56(3 \mathrm{H}, \mathrm{s}), 6.71(1 \mathrm{H}, \mathrm{s}), 6.82(1 \mathrm{H}, \mathrm{d}, J=8.4 \mathrm{~Hz})$, $6.98-7.01(1 \mathrm{H}, \mathrm{m}), 7.34-7.37(2 \mathrm{H}, \mathrm{m}), 7.55(1 \mathrm{H}, \mathrm{t}, J=8.4 \mathrm{~Hz}), 7.81-$ $7.85(2 \mathrm{H}, \mathrm{m}), 12.61(1 \mathrm{H}, \mathrm{s})$. MS m/z: $284\left(\mathrm{M}^{+}\right), 148$. Anal. Calcd for $\mathrm{C}_{16} \mathrm{H}_{12} \mathrm{O}_{3} \mathrm{~S}: \mathrm{C}, 67.60 ; \mathrm{H}, 4.25$. Found: $\mathrm{C}, 67.30 ; \mathrm{H}, 4.20$.

5-Hydroxy-4'-dimethylaminoflavone 3g Yellow crystals; mp 184.0$185.0^{\circ} \mathrm{C} .{ }^{1} \mathrm{H}-\mathrm{NMR} \delta: 3.09(6 \mathrm{H}, \mathrm{s}), 6.59(1 \mathrm{H}, \mathrm{s}), 6.75(3 \mathrm{H}, \mathrm{t}, J=8.1 \mathrm{~Hz})$, $6.96(1 \mathrm{H}, \mathrm{d}, J=8.1 \mathrm{~Hz}), 7.49(1 \mathrm{H}, \mathrm{d}, J=9.5 \mathrm{~Hz}), 7.80(2 \mathrm{H}, \mathrm{d}, J=8.9 \mathrm{~Hz})$, $12.87(1 \mathrm{H}, \mathrm{s})$. MS m/z: $281\left(\mathrm{M}^{+}\right)$, 207. Anal. Calcd for $\mathrm{C}_{17} \mathrm{H}_{15} \mathrm{NO}_{3}: \mathrm{C}$, $72.58 ; \mathrm{H}, 5.37 ; \mathrm{N}, 4.98$. Found: C, 72.46; H, 5.41; N, 5.00.

5-Hydroxy-4'-phenylflavone $3 \mathbf{h}^{17)}$ Pale yellow crystals; mp 177.0 $178.0^{\circ} \mathrm{C} .{ }^{1} \mathrm{H}-\mathrm{NMR} \delta: 6.78(1 \mathrm{H}, \mathrm{s}), 7.01(1 \mathrm{H}, \mathrm{d}, J=10.8 \mathrm{~Hz}), 7.37-7.57$ $(4 \mathrm{H}, \mathrm{m}), 7.64(2 \mathrm{H}, \mathrm{d}, J=8.1 \mathrm{~Hz}), 7.65(2 \mathrm{H}, \mathrm{d}, J=8.1 \mathrm{~Hz}), 7.98(2 \mathrm{H}, \mathrm{d}, J=$ $8.1 \mathrm{~Hz}), 12.57(1 \mathrm{H}, \mathrm{s})$. MS $m / z: 314\left(\mathrm{M}^{+}\right), 178$.

5-Hydroxy-4'-tert-butylflavone $\mathbf{3 j}^{18)}$ Yellow crystals; mp 155.0$156.0^{\circ} \mathrm{C} .{ }^{1} \mathrm{H}-\mathrm{NMR} \delta: 1.37(9 \mathrm{H}, \mathrm{s}), 6.72(1 \mathrm{H}, \mathrm{s}), 6.82(1 \mathrm{H}, \mathrm{d}, J=8.4 \mathrm{~Hz})$, $7.00(1 \mathrm{H}, \mathrm{d}, J=7.8 \mathrm{~Hz}), 7.52-7.58(3 \mathrm{H}, \mathrm{m}), 7.86(2 \mathrm{H}, \mathrm{d}, J=8.4 \mathrm{~Hz}), 12.63$ (1H, s). MS $m / z: 294\left(\mathrm{M}^{+}\right), 279$.

5-Hydroxyflavone $\left.3 \mathbf{k}^{9}\right)$ Yellow crystals; mp $145.0-146.0^{\circ} \mathrm{C} .{ }^{1} \mathrm{H}-\mathrm{NMR}$ $\delta: 6.75(1 \mathrm{H}, \mathrm{s}), 6.83(1 \mathrm{H}, \mathrm{d}, J=8.1 \mathrm{~Hz}), 7.01(1 \mathrm{H}, \mathrm{d}, J=8.6 \mathrm{~Hz}), 7.53-7.59$ $(4 \mathrm{H}, \mathrm{m}), 7.90-7.94(2 \mathrm{H}, \mathrm{m}), 12.57(1 \mathrm{H}, \mathrm{s})$. MS $m / z: 238\left(\mathrm{M}^{+}\right), 136,108$.

5-Hydroxy-4'-aminoflavone 31 Yellow crystals; mp $195.0-196.0^{\circ} \mathrm{C}$. ${ }^{1} \mathrm{H}-\mathrm{NMR} \delta: 4.13-4.23(2 \mathrm{H}, \mathrm{m}), 6.60(1 \mathrm{H}, \mathrm{s}), 6.77(3 \mathrm{H}, \mathrm{t}, J=8.5 \mathrm{~Hz}), 6.96$ $(1 \mathrm{H}, \mathrm{d}, J=8.1 \mathrm{~Hz}), 7.51(1 \mathrm{H}, \mathrm{t}, J=8.5 \mathrm{~Hz}), 7.75(2 \mathrm{H}, \mathrm{d}, J=8.6 \mathrm{~Hz}), 12.78$ $(1 \mathrm{H}, \mathrm{s})$. MS $m / z: 253\left(\mathrm{M}^{+}\right), 207,117$.

5-Hydroxy-4' $-N, N$-diacetylaminoflavone $3 \mathrm{~m}$ Yellow crystals; $\mathrm{mp}$ $202.0-203.0^{\circ} \mathrm{C} .{ }^{1} \mathrm{H}-\mathrm{NMR} \delta$ : $2.05(3 \mathrm{H}, \mathrm{s}), 2.30(3 \mathrm{H}, \mathrm{s}), 6.72(1 \mathrm{H}, \mathrm{s}), 6.82$ $(1 \mathrm{H}, \mathrm{d}, J=8.1 \mathrm{~Hz}), 7.00(1 \mathrm{H}, \mathrm{d}, J=8.1 \mathrm{~Hz}), 7.56(1 \mathrm{H}, \mathrm{t}, J=8.1 \mathrm{~Hz}), 7.63-$ $7.68(2 \mathrm{H}, \mathrm{m}), 7.92-7.97(2 \mathrm{H}, \mathrm{m}), 12.51(1 \mathrm{H}, \mathrm{s}) . \mathrm{MS} m / z: 295+42\left(\mathrm{M}^{+}\right)$, 253,117 .

5-Hydroxy-4' $\mathrm{N}$-acetylaminoflavone 3n Yellow crystals; mp 230.0 $231.0^{\circ} \mathrm{C} .{ }^{1} \mathrm{H}-\mathrm{NMR} \delta: 2.24(3 \mathrm{H}, \mathrm{s}), 6.70(1 \mathrm{H}, \mathrm{s}), 6.82(1 \mathrm{H}, \mathrm{d}, J=8.1 \mathrm{~Hz})$, $7.00(1 \mathrm{H}, \mathrm{d}, J=8.1 \mathrm{~Hz}), 7.31(1 \mathrm{H}, \mathrm{s}), 7.55(1 \mathrm{H}, \mathrm{t}, J=8.1 \mathrm{~Hz}), 7.70(2 \mathrm{H}, \mathrm{d}$, $J=8.1 \mathrm{~Hz}), 7.90(2 \mathrm{H}, \mathrm{d}, J=8.1 \mathrm{~Hz}), 12.61(1 \mathrm{H}, \mathrm{s}) . \mathrm{MS} m / z: 295\left(\mathrm{M}^{+}\right), 253$,
207

5,4'-Difluoroflavone 7a Colorless crystals; mp $190.0-191.0{ }^{\circ} \mathrm{C} .{ }^{1} \mathrm{H}-$ NMR $\delta$ : $6.71(1 \mathrm{H}, \mathrm{s}), 7.05-7.12(1 \mathrm{H}, \mathrm{m}), 7.20-7.26(2 \mathrm{H}, \mathrm{m}), 7.37(1 \mathrm{H}, \mathrm{d}$, $J=8.6 \mathrm{~Hz}), 7.60-7.67(1 \mathrm{H}, \mathrm{m}), 7.88-7.94(2 \mathrm{H}, \mathrm{m}) . \mathrm{MS} m / z: 258\left(\mathrm{M}^{+}\right)$, 110. Anal. Calcd for $\mathrm{C}_{15} \mathrm{H}_{8} \mathrm{O}_{2} \mathrm{~F}_{2}: \mathrm{C}, 69.77 ; \mathrm{H}, 3.12$. Found: $\mathrm{C}, 69.56 ; \mathrm{H}$, 3.21 .

5-Fluoro-4' -chloroflavone $7 \mathrm{~b}$ Colorless crystals; mp 195.0-196.0 ${ }^{\circ} \mathrm{C}$. ${ }^{1} \mathrm{H}-\mathrm{NMR} \delta: 6.74(1 \mathrm{H}, \mathrm{s}), 7.05-7.12(1 \mathrm{H}, \mathrm{m}), 7.38(1 \mathrm{H}, \mathrm{d}, J=8.6 \mathrm{~Hz})$, $7.50-7.53(2 \mathrm{H}, \mathrm{m}), 7.60-7.68(1 \mathrm{H}, \mathrm{m}), 7.83-7.86(2 \mathrm{H}, \mathrm{m}), \mathrm{MS} m / z: 274$ $\left(\mathrm{M}^{+}\right), 138,110$

5-Fluoro-4'-cyanoflavone 7c Colorless crystals; $\mathrm{mp} 201.8-202.0^{\circ} \mathrm{C}$. ${ }^{1} \mathrm{H}-\mathrm{NMR} \delta$ : $6.81(1 \mathrm{H}, \mathrm{s}), 7.08-7.15(1 \mathrm{H}, \mathrm{m}), 7.38-7.42(1 \mathrm{H}, \mathrm{m}), 7.63-$ $7.72(1 \mathrm{H}, \mathrm{m}), 7.82-7.85(2 \mathrm{H}, \mathrm{m}), 8.00-8.04(2 \mathrm{H}, \mathrm{m}) . \mathrm{MS} m / z: 265\left(\mathrm{M}^{+}\right)$, $237,138,110$

5-Methoxy-4'-fluoroflavone $\mathbf{1 0 a}^{20)}$ Pale yellow crystals; mp 149.0$150.0^{\circ} \mathrm{C} .{ }^{1} \mathrm{H}-\mathrm{NMR} \delta: 4.01(3 \mathrm{H}, \mathrm{s}), 6.68(1 \mathrm{H}, \mathrm{s}), 6.84(1 \mathrm{H}, \mathrm{d}, J=8.1 \mathrm{~Hz})$, $7.11-7.23(3 \mathrm{H}, \mathrm{m}), 7.58(1 \mathrm{H}, \mathrm{t}, J=8.1 \mathrm{~Hz}), 7.87-7.94(2 \mathrm{H}, \mathrm{m})$. Anal. Calcd for $\mathrm{C}_{16} \mathrm{H}_{11} \mathrm{O}_{3} \mathrm{~F}$ : C, 71.11; H, 4.10. Found: $\mathrm{C}, 71.35 ; \mathrm{H}, 4.26$.

5-Methoxy-4'-trifluoromethylflavone $\mathbf{1 0 b}^{\mathbf{2 0}} \quad$ Yellow crystals; mp 171.0$172.0{ }^{\circ} \mathrm{C} .{ }^{1} \mathrm{H}-\mathrm{NMR} \delta: 4.02(3 \mathrm{H}, \mathrm{s}), 6.79(1 \mathrm{H}, \mathrm{s}), 6.86(1 \mathrm{H}, \mathrm{d}, J=8.1 \mathrm{~Hz})$ $7.16(1 \mathrm{H}, \mathrm{d}, J=10.8 \mathrm{~Hz}), 7.61(1 \mathrm{H}, \mathrm{t}, J=8.1 \mathrm{~Hz}), 7.78(2 \mathrm{H}, \mathrm{d}, J=8.1 \mathrm{~Hz})$, $8.02(2 \mathrm{H}, \mathrm{d}, J=8.1 \mathrm{~Hz})$, Anal. Calcd for $\mathrm{C}_{17} \mathrm{H}_{11} \mathrm{O}_{3} \mathrm{~F}_{3}: \mathrm{C}, 63.75 ; \mathrm{H}, 3.46$. Found: C, 63.49; H, 3.60.

Acknowledgements This research was partially supported by the Ministry of Education, Culture, Sports, Science and Technology, Grant-in-Aid for Scientific Research (C), 20580115, and the fund for Agriomics project. We thank Prof. Shuji Akai, Shizuoka Pharmaceutical University, for the elemental analysis. We also thank The Uehara Memorial Foundation for funding this work.

\section{References and Notes}

1) Cohen M. B., Rokhlin O. W., J. Cell. Biochem., 3, 363-371 (2006).

2) Mooradian A. D., Morley J. E., Korenman S. G., Endocr. Rev., 8, 128 (1987)

3) Kao Y. C., Zhou C., Sherman M., Laughton C. A., Chen S., Environ. Health Perspect., 106, 85-92 (1998).

4) Bovee Toine F. H., Schoonen Willem G. E. J., Hamers Astrid R. M., Bento Marta J., Peijnenburg Ad A. C. M., Anal. Bioanal. Chem., 390, 1111-1119 (2008)

5) Nishizaki Y., Ishimoto Y., Hotta Y., Hosoda A., Yoshikawa H., Akamatsu M., Tamura H., Bioorg. Med. Chem. Lett., 19, 4706-4710 (2009).

6) Hammett L. P., Chem. Rev., 17, 125-136 (1935)

7) Hansch C., Leo A., Unger S. H., Kim K. H., Nikaitani D., Lien E. J., J. Med. Chem., 16, 1207-1216 (1973).

8) Hansch C., Rockwell S. D., Jow P. Y. C., Leo A., Steller E. E., J. Med. Chem., 20, 304-306 (1977).

9) Bois F., Beney C., Mariotte A., Boumendjel A., Synlett, 9, 14801482 (1999).

10) Typical Experimental Procedure; Preparation of 5-hydroxy-4'-fluoroflavone 3a: To a stirred suspension of 2,6-dihydroxyacetophenone 1a $(229 \mathrm{mg}, 1.5 \mathrm{mmol})$ and 4 -fluorobenzoyl chloride $2 \mathrm{a}(357 \mathrm{mg}, 2.25$ $\mathrm{mmol})$ in acetone $(10 \mathrm{ml})$, was added potassium carbonate $(2.1 \mathrm{~g}, 15.2$ $\mathrm{mmol}$ ) at room temperature. The reaction mixture was stirred at $70^{\circ} \mathrm{C}$ for $24 \mathrm{~h}$, and quenched with $1 \mathrm{M} \mathrm{HCl}$, then extracted with AcOEt. The organic layer was washed with brine, dried with sodium sulfate and concentrated in vacuo. The residue was purified by recrystallization from ethyl acetate $/ n$-hexane to give 5 -hydroxy-4'-fluoroflavone 3a ( $86 \mathrm{mg}, 22 \%$ yield) as yellow crystals.

11) 5-Hydroxy-3-(4-methylbenzoyl)-2-p-tolyl-4H-chromen-4-one $\quad(\mathbf{4 i})^{16)}$ : Pale yellow crystals; mp $223.0-224.0{ }^{\circ} \mathrm{C} .{ }^{1} \mathrm{H}-\mathrm{NMR} \delta: 2.34(3 \mathrm{H}, \mathrm{s})$, $2.39(3 \mathrm{H}, \mathrm{s}), 6.85(1 \mathrm{H}, \mathrm{d}, J=8.1 \mathrm{~Hz}), 7.02(1 \mathrm{H}, \mathrm{d}, J=7.6 \mathrm{~Hz}), 7.15-$ $7.26(4 \mathrm{H}, \mathrm{m}), 7.53-7.63(3 \mathrm{H}, \mathrm{m}), 7.84(2 \mathrm{H}, \mathrm{d}, J=8.4 \mathrm{~Hz}), 12.23(1 \mathrm{H}$, s). MS $m / z: 370\left(\mathrm{M}^{+}\right)$, 341. 2-(4-tert-Butylphenyl)-5-hydroxy-3-(4methylbenzoyl)-4H-chromen-4-one (4j): Pale yellow crystals; $\mathrm{mp}$ $228.0-229.0^{\circ} \mathrm{C} .{ }^{1} \mathrm{H}-\mathrm{NMR} \delta: 1.28(9 \mathrm{H}, \mathrm{s}), 1.33(9 \mathrm{H}, \mathrm{s}), 6.85(1 \mathrm{H}, \mathrm{d}$, $J=8.4 \mathrm{~Hz}), 7.02(1 \mathrm{H}, \mathrm{d}, J=8.6 \mathrm{~Hz}), 7.39(2 \mathrm{H}, \mathrm{d}, J=7.8 \mathrm{~Hz}), 7.47(2 \mathrm{H}$, d, $J=8.6 \mathrm{~Hz}), 7.59-7.61(3 \mathrm{H}, \mathrm{m}), 7.90(2 \mathrm{H}, \mathrm{d}, J=8.4 \mathrm{~Hz}), 12.22(1 \mathrm{H}$, s). MS m/z: $454\left(\mathrm{M}^{+}\right), 397,207$. Anal. Calcd for $\mathrm{C}_{30} \mathrm{H}_{32} \mathrm{O}_{4}$ : C, 78.92; H, 7.06. Found: C, 78.73; H, 6.58 .

12) Moodie R. B., Schofield K., Accounts of Chemical Research, 9, 287292 (1976)

13) Kagawa H., Shigematsu A., Ohta S., Harigaya Y., Chem. Pharm. Bull., 
53, 547-554 (2005).

14) Dao T. T., Chi Y. S., Kim J., Kim H. P., Kim S., Park H., Bioorg. Med. Chem. Lett., 14, 1165-1167 (2004).

15) Ares J. J., Outt P. E., Kakodkar S. V., Buss R. C., Geiger J. C., J. Org. Chem., 58, 7903-7905 (1993).

16) Pinto D. C. G. A., Silva A. M. S., Almeida L. M. P. M., Cavaleiro J. A. S., Elguero J., Eur. J. Org. Chem., 22, 3807-3815 (2002).

17) Baston D. S., Nagy S. R., Nantz M., Kurth M., Springsteel M., Denison M. S., Organohalogen Compounds, 65, 142-145 (2003).

18) Beutner S., Frixel S., Ernst H., Haremza S., Hoffmann T., Hernandez-
Blanco I., Kiesendahl N., Mayer B., Noack P., Ruck C., Scherrers R., Schmidt M., Schuelke I., Martin H. D., Advances in Colour Science and Technology, 5, 103-112 (2002).

19) Fitzmaurice R. J., Etheridge Z. C., Jumel E., Woolfson D. N., Caddick S., Chem. Commun., 46, 4814-4816 (2006).

20) Ares J. J., Outt P. E., Randall J. L., Murray P. D., Weisshaar P. S., O’Brien L. M., Ems B. L., Kakodkar S. V., Kelm G. R., Kershaw W. C., Werchowski K. M., Parkinson A., J. Med. Chem., 38, 4937-4943 (1995). 